\title{
Política de discapacidad e inclusión de la Universidad del Valle: un proceso participativo
}

\author{
Política de discapacidad e inclusión at \\ Universidad del Valle: A Participatory Process
}

\section{Política de discapacidad e inclusión da Universidad del Valle: um processo participativo}

Mónica María Carvajal Osorio ${ }^{1}$

Profesora e investigadora de la Universidad del Valle, Cali, Colombia monica.carvajal@correounivalle.edu.co 


\title{
Resumen
}

La población con discapacidad es uno de los grupos que se encuentran en riesgo social al exponerse a situaciones de exclusión en diversos ámbitos de la vida ciudadana, entre estos el contexto universitario. La Universidad del Valle, en el proceso de reconocer la discapacidad, emprendió la construcción de la Política de discapacidad e inclusión con la participación de actores de la comunidad universitaria. La sistematización a través del análisis de fuentes documentales muestra tres características del proceso: carácter participativo y de corresponsabilidad, sentido formativo, y trabajo en red. Se espera aportar en la orientación de experiencias similares por su valor en la adopción de una identidad colectiva para el desarrollo institucional: "re-conocer" la discapacidad y a las personas con discapacidad.

Palabras clave: inclusión; equidad; participación; personas con discapacidad.

\begin{abstract}
The population with disabilities is one of the groups at social risk of exclusion within different citizen's life settings including higher education. Universidad del Valle, in the process of recognizing the disability, undertook the construction of Política de discapacidad e inclusión with the participation of diverse actors from the University community. Through the analysis of documentary sources, this systematization describes three main characteristics of the process: participative character and joint responsibility of actors, formative approach and networking. Results of this study will contribute to guide the design of other disability policies and constitutes a valuable experience focusing on the construction of community identity for institutional development: "recognition" of disability and persons with disability.
\end{abstract}

Keywords: inclusion; equity; participation; persons with disability.

\section{Resumo}

A população com deficiência é um grupo que se encontra em risco social, pois fica exposto a situações de exclusão em diversos âmbitos da vida cidadã, dentre eles o contexto universitário. A Universidad del Valle, no processo de reconhecer as pessoas portadoras de deficiência, iniciou a construção da Política de discapacidad e inclusión, com a participação de atores da comunidade universitária. A sistematização através da análise de fontes documentais, expressa três características do processo: caráter participativo e de corresponsabilidade, sentido formativo, e trabalho em rede. O objetivo é aportar na orientação de experiências similares pelo seu valor na adoção de uma identidade coletiva para o desenvolvimento institucional: "re-conhecer" a deficiência e as pessoas portadoras de deficiência.

Palavras-chave: inclusão; equidade; participação; pessoas portadoras de deficiência.

\footnotetext{
¿Cómo citar este artículo? / How to quote this article?

Carvajal, Mónica. «Política de discapacidad e inclusión de la Universidad del Valle: un proceso participativo». sociedad y economía, $\mathrm{n}^{\circ} 29$ (julio - diciembre 2015): 175-201.
} 


\section{Introducción}

En la historia de la humanidad han hecho presencia diferentes paradigmas que han marcado las concepciones dadas en torno a la discapacidad y a las personas con discapacidad desde modelos que comprenden y explican este asunto desde lo mitológico, lo religioso y lo biológico hasta planteamientos enfocados en el fenómeno de la discapacidad como un asunto social, así como una cuestión de derechos y de autonomía personal (Díaz 2009; Ferreira 2008; Palacios y Bariffi 2007). La evolución en las formas de comprender la discapacidad ha tenido una influencia positiva sobre el reconocimiento de las personas con discapacidad como sujetos de derechos. De ahí que la presencia de políticas sociales dirigidas a esta población en Colombia se haya hecho evidente después de la Constitución Política de 1991.

La Universidad del Valle, institución de educación superior de carácter público, ubicada en Santiago de Cali, en el suroccidente colombiano, ha hecho su camino para reconocer la presencia de las personas con discapacidad en su entorno. La construcción y adopción de una política se constituyó en una meta y en un medio para la exhortación al posicionamiento de dos asuntos que deben comprenderse y abordarse articuladamente en el contexto de la vida universitaria: la discapacidad y la inclusión. En este proceso, se puso en marcha la discusión con participación de diferentes sectores de la comunidad universitaria, integrada por personas con y sin discapacidad hasta incidir en la agenda institucional.

El presente artículo recoge evidencias de dicho proceso de construcción en el período comprendido entre el 2003 y el 2012, el cual estuvo nutrido desde las formas de comprender la discapacidad bajo el enfoque biopsicosocial (OMS 2001) y el social (Díaz 2009; Ferreira 2008; Palacios y Bariffi 2007). La sistematización del proceso se logró a través de fuentes documentales elaboradas durante el proceso de construcción de la Política de discapacidad e inclusión de la Universidad del Valle (Universidad del Valle 2013): proyecto de práctica de estudiantes de último año de fonoaudiología de la Escuela de Rehabilitación Humana, relatorías, actas, instrumentos institucionales, así como documentos de trabajo, e informes técnicos y de investigación formativa articulados al proceso. De la recuperación de la experiencia y su análisis emergieron tres características del proceso: carácter participativo en la construcción de la política con principio de corresponsabilidad; sentido formativo y de construcción en espiral; y estrategia de trabajo en red. Estas surgieron del análisis del proceso reconstruido a la luz de los desarrollos conceptuales en torno a la construcción de políticas públicas en discapacidad (CPPS y CAF 2002; Otoya, Quintana y Tenorio 2003), los paradigmas y modelos para comprender la discapacidad, la actuación social de las personas con discapacidad, así como los aportes sobre justicia social y discapacidad (Sen 2004; Nussbaum 2006).

En el desarrollo del presente artículo, en primera instancia se brinda un panorama general que ubica algunos elementos que ponen en evidencia la situación de desigualdad que vive la población con discapacidad internacional y nacionalmente; esto se muestra particularmente en los ámbitos educativo y laboral al tomar como base que la población que habita el campus universitario desempeña, 
predominantemente, dos roles: ser estudiante y/o ser funcionario (docente o no docente). Luego, se mira a la universidad como institución social que aporta a la instalación de condiciones para contrarrestar las inequidades a las que se enfrenta la población con discapacidad.

Con estos elementos, se aborda la reconstrucción de la experiencia para el diseño y adopción de la Política de discapacidad e inclusión de la Universidad del Valle, Acuerdo 004 del 12 de julio de 2013 (Universidad del Valle 2013). Se muestra la forma adoptada desde su carácter participativo, corresponsable y formativo donde la acción colectiva fue protagonista al convocar diversos actores integrantes de la comunidad universitaria que se sintieron identificados con el asunto. Por último, se ofrecen algunas conclusiones que resaltan el valor de la participación de las personas con discapacidad en la configuración de su entorno como potenciador de sus capacidades y realización personal.

\section{La población con discapacidad. Grupo en riesgo social}

La población con discapacidad es uno de los grupos que se encuentran en mayor riesgo social al exponerse a situaciones de exclusión en los diversos ámbitos de la vida ciudadana. Esta realidad ha propiciado, históricamente, una mirada particular desde organismos internacionales y desde los Estados para analizar la realidad a la que se enfrenta en los diferentes entornos y diseñar respuestas culturalmente acordes con los requerimientos de desarrollo.

Una aproximación a esta realidad en lo referido a cifras la ofrece el Informe mundial sobre la discapacidad de 2011, al indicar que más de mil millones de personas viven con algún tipo de discapacidad, representando, aproximadamente, $15 \%$ de la población mundial al 2010 (OMS y BM 2011). En el caso de América Latina, en el 2006 se estimó que 85 millones de personas expresan alguna forma de discapacidad (OMS y BM 2011). En Colombia, a partir de los datos del Censo 2005, se identificaron 2.765 .491 personas con discapacidad, con una prevalencia de 6,3\%, y particularmente en el Valle del Cauca la prevalencia se estimó en 6,7 $\%$ (DANE 2005).

En lo concerniente a la vivencia de exclusión, esta se traduce en mayores dificultades de acceso a los recursos que configuran la cotidianidad de todo ciudadano como son los servicios sanitarios, educativos, laborales, de transporte, ocio, recreación y deporte (OMS y BM 2011; DANE 2005). Este hecho lleva a plantear la discapacidad como una experiencia con fuertes implicaciones sociales y económicas tanto para la población misma como para sus familias, comunidades y naciones, como se evidencia en diferentes informes que se han adelantado. El Informe de seguimiento de la educación EPT en el mundo. Llegar a los marginados (Unesco y Ministerio de Educación y Ciencia de España 2010) indica que aproximadamente 75 millones de niños no pueden ejercer su derecho a la educación, siendo una de las causas principales de dicha exclusión presentar una condición de discapacidad. En la misma línea, el Informe mundial sobre la discapacidad (OMS y BM 2011) indica que en los 51 países incluidos en el análisis de la aplicación de la encuesta mundial de salud, esta población registra una tasa de terminación de la escuela primaria significativamente más baja y un 
promedio de años de educación menor en relación con las personas sin discapacidad (hombres: 5,96 años, en comparación con 7,03 años, respectivamente; mujeres: 4,98 años, en comparación con 6,26 años, respectivamente). El análisis de dicha encuesta revela que las personas con discapacidad, en general, viven la experiencia de la desigualdad en la atención en salud y tienen más necesidades insatisfechas; igualmente, no cuentan con un pleno acceso a servicios de apoyo, no se les brinda mecanismos de información y comunicación accesible, ni sus entornos cuentan con ambientes físico-arquitectónicos favorables (OMS y BM 2011).

En lo referido a trabajo y empleo, dicho informe expresa que las personas con discapacidad, en edad de trabajar, enfrentan considerables desventajas y peores resultados en el mercado de trabajo que las personas sin discapacidad en edad de trabajar, así como tasas de ocupación más bajas. Un estudio de la Organización para la Cooperación y Desarrollo Económicos (2010) en 27 países, así lo precisa:

En 27 países, las personas con discapacidad en edad de trabajar, en comparación
con sus homólogas no discapacitadas, experimentaban desventajas significativas en
el mercado laboral y tenían peores oportunidades de empleo. En promedio, su tasa
de empleo (44\%) era ligeramente superior a la mitad de la de las personas sin dis-
capacidad (75\%). La tasa de inactividad era unas 2,5 veces mayor entre las personas
sin discapacidad (OECD 2010 en OMS y BM 2011, 269).

En el plano nacional, los reportes guardan una similitud en su comportamiento. El DANE (2005) planteó que de 6,3\% de personas que cuentan con una limitación permanente, $33,3 \%$ no han accedido a ningún nivel educativo, 29,1 \% cuentan con básica primaria incompleta, 2,34\% tiene algún nivel de educación superior (técnica, tecnológica, profesional) hasta llegar a plantear un escaso $1 \%$ de población con discapacidad que logra culminar la educación superior y un escaso $0,1 \%$ que alcanza la formación posgraduada. Esto significa, como lo plantea el Consejo Nacional de Política Económica y Social (DNP y CONPES 2013), que el nivel educativo de las personas con discapacidad es mucho menor que el del resto de la población. De otra parte, en el plano laboral se muestra que, $14 \%$ de la población colombiana con discapacidad, registrada, tiene empleo. Del $86 \%$ restante, se resalta que $31,8 \%$ no trabaja y no cuenta con pensión, $25 \%$ se dedica a oficios domésticos, 3,2\% realiza actividades de autoconsumo y $1,7 \%$ es pensionado (MPS 2011 en DNP y CONPES 2013).

Así pues, la visibilización de la realidad de esta población internacional, nacional y localmente, ha demandado medidas de Estado para conducir a una mejor vivencia de ser ciudadano donde se logre habitar, sin restricciones, los diversos espacios que culturalmente se disponen para el desarrollo de todo ser humano. No obstante, las respuestas que se configuran para contrarrestar la realidad que experimentan las personas con discapacidad en las diferentes esferas de su vida, están mediadas por las formas de comprender la discapacidad, hecho que plantea diversidad de respuestas. 


\section{Políticas de discapacidad en el marco del reconocimiento de los derechos de las personas con discapacidad}

Como lo muestran los anteriores informes, aún no logra ser una realidad experimentada por todas las personas con discapacidad su reconocimiento como ciudadanos con los mismos derechos y deberes al lado de los demás colombianos, con capacidad de elegir y de aportar al desarrollo social del país. Una de las razones se centra en las formas de comprender la discapacidad.

Justamente, por ser la discapacidad un fenómeno o condición tan antigua como el hombre (Pantano 2009), el carácter diverso y cambiante en su concepción y en el diseño de las respuestas sociales que se desprenden muestra un transitar de un modelo médico centrado en la deficiencia en las estructuras y funciones corporales como el factor determinante de las posibilidades de realización humana hacia un modelo social que resalta el valor de la autonomía y de las diferencias, en el cual las estructuras sociales, políticas y económicas definen las condiciones de participación y desarrollo de las personas con discapacidad. Desde este último modelo, el entorno es el que "discapacita" y, por tanto, el que genera o consolida la exclusión de estas personas (Díaz 2009). La comprensión del impacto que tiene el entorno en la configuración de situaciones de exclusión abrió un panorama hacia el reconocimiento de los derechos y libertades fundamentales de las personas con discapacidad como un asunto que debe ser promovido y protegido. Por tanto, el modelo social de la discapacidad ha contribuido en la instalación de una perspectiva de derechos frente a la cual organismos internacionales, Estados e instituciones se enfrentan a la tarea de diseñar respuestas garantistas. Las respuestas que se esperan desde este modelo, sin negar la condición biológica de dichos sujetos, plantean una responsabilidad individual y colectiva que confluye en las modificaciones contextuales necesarias para que dicha población participe en igualdad de condiciones en las diferentes esferas de la vida ciudadana.

Sin embargo, como lo plantean Jiménez y Huete (2010), la presencia del modelo social no niega la coexistencia con los otros modelos fundamentados en principios eugenésicos, de marginación, o de explicaciones biológicas que generan respuestas sociales marcadas por formas de eliminación bajo el amparo del desarrollo de la ciencia y la biotecnología, formas de discriminación que anulan las capacidades de los sujetos y la insuficiente provisión de servicios de rehabilitación funcional en el contexto de un sistema de salud que rompe con los desarrollos conceptuales en torno a la preservación de la salud. Por ello, en el camino de enfrentar el problema del reconocimiento de los derechos de las personas con discapacidad, es pertinente, como lo plantea Parra y Palacios (2007), identificar el concepto de discapacidad que emerge en el seno de las comunidades, para luego desarrollarlo y relacionarlo con el ambiente en el que se inscribe la discusión con el fin de lograr un acercamiento a la dimensión de la situación que se presenta.

Reconocer los derechos sustanciales que tiene toda persona con discapacidad para alcanzar un "estar" y un "ser" sin menoscabar su dignidad implica el reconocimiento del derecho a la igualdad en la diferencia y esta, a su vez, requiere 
ser reconocida como una condición del ser humano. De acuerdo con los planteamientos de Rawls (1971, en Moreno 2011), reconocer la diferencia es un principio articulado a la libertad que debe estar en la base de toda institución básica, y esta es una aspiración elemental de todo grupo de individuos que ocupan un lugar en la sociedad. A pesar de que los derechos fundamentales para todas las poblaciones fueron declarados desde 1948 (ONU 1948) y están planteados desde un principio de reconocimiento a la diversidad, históricamente se ha insistido en el diseño de marcos específicos que reiteren los derechos no solo de poblaciones con discapacidad, sino de todos aquellos a quienes se les vulneran sus libertades: grupos cuyo origen cultural, étnico, de preferencia sexual, socioeconómico y condición de discapacidad, entre otros, luchan por el acceso a condiciones dignas para su realización humana.

En medio de los diferentes paradigmas que coexisten en torno a la discapacidad y a las personas con discapacidad, el Estado colombiano dio un paso adelante al plantear en la Constitución Política de 1991, en su Artículo 13, el reconocimiento de las obligaciones hacia las personas con discapacidad, sus derechos legales, la igualdad y la garantía para su goce sin discriminación. El soporte constitucional impulsó el inicio de acciones de política pública nacional (DNP y CONPES 2004; 2013) que posibilitó la ganancia de espacios en las agendas gubernamentales con impacto regional y local desde principios del siglo XXI. La construcción de respuestas sociales para la discapacidad desde el diseño de políticas públicas específicas para este grupo poblacional trajo consigo un cambio de actitud en la relación de la sociedad civil con el Estado.

La primera experiencia que tuvo Colombia de trabajo participativo entre sociedad civil y sector gubernamental en torno a la discapacidad fue en la formulación participativa de la política de discapacidad en el período 2001-2002 con la participación de los departamentos del Atlántico, Caldas y Valle (CPPS y CAF 2002). Este proceso, planteado de manera descentralizada, demandó la búsqueda de mecanismos de coordinación y concertación entre los sectores, las instituciones y la sociedad civil, lo cual promovió y exigió la participación desde una expectativa de constituir espacios de formación de personas y grupos con discapacidad concebidos a sí mismos como sujetos de derechos, capaces de contribuir a las decisiones públicas desde su red de experiencias y vivencias cotidianas (CPPS y CAF 2002). Esta nueva forma de actuación en torno a la discapacidad y a las personas con discapacidad fue instalando en el país un sistema de autoorganización a partir de la búsqueda de nuevas relaciones y formas de actuación tanto para proponer y llegar a consensos como para asumir veeduría sobre sus propias iniciativas (CPPS y CAF 2002).

En este plano de la construcción de política pública de discapacidad, el municipio de Cali movilizó un proceso participativo en el 2002. Se resaltó el lugar de la corresponsabilidad al evidenciar la respuesta de la sociedad en la contribución para que todas las personas que hacen parte de ella ocupen el lugar que les corresponde, asuman sus deberes y hagan pleno uso de sus derechos (Otoya, Quintana y Tenorio 2003). Para ello, se enfatizó en la necesidad de la acción interrelacionada de la persona con discapacidad y la sociedad.

En la construcción de esta política se insistió, además, en la consideración de todos aquellos obstáculos que impiden que una persona con discapacidad 
satisfaga las necesidades fundamentales del ser, hacer, tener y estar en la sociedad (Otoya, Quintana y Tenorio 2003). Esto fundamentado en que hasta que la persona con discapacidad no encuentre su nicho en su entorno y la sociedad no le dé un trato con equidad, no se puede hablar de construcción de tejido social ni de actuación con equidad. Por ello, este proceso municipal contó con voces de diferentes actores e instancias de la sociedad caleña: personas con y sin discapacidad, la academia, organizaciones de y para personas con discapacidad, Estado, familias, profesionales que brindan servicios de diverso orden a las personas con discapacidad, entre otros.

La definición de política pública que se adoptó para este proceso de construcción resalta el valor de la colectividad para orientar un cambio en la sociedad:

\footnotetext{
Un conjunto conformado por uno o varios objetivos colectivos considerados necesarios o deseables, por medios y acciones, que son tratados, por lo menos parcialmente, por una institución u organización gubernamental con la finalidad de orientar el comportamiento de actores individuales o colectivos para edificar una situación percibida como insatisfactoria o problemática (Roth 2006, 27).
}

Las características que le dieron identidad al diseño de la política en el contexto municipal son evidencia de la concreción lograda a partir de la definición planteada por Roth (2006). Este fue un proceso participativo y concertado, incluyente, formativo e investigativo, prospectivo y sostenible. Articulado con estas características, es significativa la presencia transversal de la relación discapacidad y sociedad al tener una consideración sobre el mismo asunto de la discapacidad; el diseño y puesta en marcha de toda la política de discapacidad no solo debe garantizar una respuesta integral para el colectivo de personas con discapacidad, sino que además debe reforzar la imagen de ellas como ciudadanos que, más que recibir, también contribuyen a la sociedad (Otoya, Quintana y Tenorio 2003).

Las políticas nacionales o locales cohesionadas con los marcos internacionales son referentes actuales que brindan cierto nivel de soporte a la modificación de las realidades que se constituyen en torno a las personas con discapacidad. Se resalta la Convención sobre los Derechos de las Personas con Discapacidad (ONU 2006), ratificada por muchos países, entre ellos Colombia a través de la Ley 1.346 de 2009 y la Ley 1.618 de 2013. En materia de educación superior, se cuenta con los Lineamientos de política de de educación superior inclusiva (MEN 2013), en los cuales se reconoce a la población con discapacidad como uno de los grupos en desventaja que históricamente no ha contado con condiciones de equidad para su desarrollo profesional.

Sin restar valor a este avance internacional y nacional, estos marcos no son suficientes si no van acompañados de procesos formativos con las comunidades, que permitan movilizar los imaginarios existentes en torno a la discapacidad, construidos desde la perspectiva del déficit. Esto exige reconocer la diferencia humana en una relación de reciprocidad con el otro que considere la diferencia como una característica esencial que marca las individualidades de todos los seres humanos y no como una condición referida exclusivamente a las personas con discapacidad. 


\section{La Universidad frente a la presencia de personas con discapacidad}

El reconocimiento del derecho a la educación de las personas con discapacidad ha estado articulado, históricamente, a las diversas formas de comprender la discapacidad. Existe un extenso número de instrumentos internacionales que orientan a los Estados e instituciones para garantizar el acceso a la educación (Unesco e IESALC 2006). No obstante, el derecho al acceso, permanencia y titulación en la educación superior no ha sido un tema explícito en dichos marcos. En su lugar, la responsabilidad de la Universidad en el panorama de la educación superior inclusiva fue considerada desde sus funciones sustantivas de formación, investigación y extensión: en el marco del evento mundial que marcó la definición de principios de la educación inclusiva, la Conferencia mundial sobre necesidades educativas especiales: acceso y calidad (Unesco y Ministerio de Educación y Ciencia de España 1994), se destacó la importancia de la Universidad como generadora de cultura, constructora de conocimientos, formadora de capacidades y valores en los profesionales y cuna de las ideas más avanzadas de transformación social. Se le otorgó a las universidades el importante papel de ser consultores y, en particular, se le asignó la responsabilidad de la investigación, la evaluación, la preparación de formadores de profesores y la elaboración de programas y materiales pedagógicos. Se destaca el énfasis dado a la participación activa de las personas con discapacidad en la investigación y formación con el fin de garantizar la consideración de sus puntos de vista (Alcantud 2004 en Unesco e IESALC 2006).

Sin negar el valor de la función otorgada a la Universidad en el escenario de una "educación para todos", la invisibilidad de las personas con discapacidad en la educación superior hasta la década de 1990 es evidente. La Universidad fue ubicada por fuera de la realidad sin que se le asignara ninguna responsabilidad en la generación de condiciones de exclusión hacia dichas personas. La Ley 30 de 1992, Ley de Educación Superior, y la Ley 115 de 1994, Ley General de Educación, son evidencias; en ninguno de sus artículos aparecen planteamientos en los que se expresen garantías a la población con discapacidad en la educación superior.

Solo en el 2006 aparecen en el panorama de Latinoamérica expresiones directas sobre el asunto, gracias al Informe sobre la educación superior en América Latina y el Caribe 2000-2005 (Unesco e IESALC 2006). En este contexto, se hace visible la demanda del derecho al acceso a la educación superior en condiciones de igualdad sobre la base del reconocimiento de la diversidad humana y, sobre todo, a la permanencia y conclusión de estudios al mostrar este escenario como el más excluyente de todos.

Este llamado no significa que antes no se tuvieran respuestas del Estado o institucionales en este nivel de formación. De hecho, la primera expresión de Universidad diseñada desde la diferencia es la Universidad de Gallaudet en Washington, creada en 1864 para estudiantes sordos. En la década de 1950, se resalta el campus de Southern Illinois University por los ajustes planteados, sumado a la actuación de otras universidades norteamericanas posterior al Rehabilitation Act, en 1973 (Sassaki 2001 en Unesco e IESALC 2006). 
Por otra parte, el sistema de las Naciones Unidas cuenta con un extenso número de declaraciones, normas y documentos (en promedio 14) que han brindado sustento a la educación superior inclusiva por más de 50 años; inicia con la Declaración Universal de los Derechos Humanos (ONU 1948) y se extiende hasta la Convención sobre los Derechos de las personas con discapacidad (ONU 2006). A esto se suma la actuación de 17 países latinoamericanos que han agenciado marcos normativos en el contexto general de la integración e inclusión de las personas con discapacidad en las últimas tres décadas, de los cuales nueve dan cuenta de iniciativas gubernamentales e institucionales favorecedoras de una educación superior abierta a la población con discapacidad: Argentina, Brasil, Chile, Colombia, Costa Rica, Ecuador, Honduras, Perú y Venezuela (Unesco e IESALC 2006). En Colombia, los estudios muestran respuestas institucionales desde el diseño de acciones específicas por iniciativa de profesores, acciones, servicios o programas institucionales (Molina 2006; Hurtado, Jaramillo y Núñez 2013).

A pesar de la extensa normativa en materia de discapacidad desarrollada en Latinoamérica, el informe de la UNESCO e IESALC (2006) hace un llamado sobre el incumplimiento de los marcos y destaca algunos factores limitantes: la ausencia de normas detalladas y específicas, su obsolescencia o inaplicabilidad; la no ratificación de convenios internacionales; el incumplimiento por parte del Estado de su función planificadora y reguladora; el desconocimiento de las leyes por parte de los actores y de los beneficiarios, así como el deficiente ejercicio de la ciudadanía por parte de las personas con discapacidad como elemento contribuyente a la reproducción de dinámicas excluyentes (Rama 2006, en Unesco e IESALC 2006). También resalta la complejidad de la situación de esta población como un asunto que suma a la historia latinoamericana, ya que al coexistir con desigualdades de otro orden se corre el riesgo de perder la particularidad de la experiencias de exclusión de la población con discapacidad:

\footnotetext{
Este hecho se hace muy peligroso en nuestra región formada por países que han coexistido históricamente (y coexisten aún) con la desigualdad económica, de género, racial y étnica; lo anterior se demuestra en políticas públicas e iniciativas que conceden mayor visibilidad a los pobres, mujeres, negros e indígenas mientras entre ellos "se pierden" las personas con discapacidad, que muy probablemente forman parte de esos grupos pero que tienen diferencias que, añadidas, los hacen aún más vulnerables (Unesco e IESALC 2006, 148-149).
}

El tipo de respuestas institucionales que se requieren para lograr mayor impacto se dirigen hacia el diseño y adopción de políticas focalizadas para esta población en el ámbito institucional, como una de las alternativas que podrían impactar estructuralmente a la Universidad hacia la reconstrucción de condiciones más dignas y que transciendan en una cultura reconocedora de la diferencia y en el fortalecimiento de prácticas más inclusivas en los diferentes espacios de la vida universitaria.

A pesar de no encontrar reportes sobre abordajes de este tipo en el panorama internacional, en el nacional se identifican las experiencias de dos universidades públicas de Colombia: Universidad Nacional (2012: Acuerdo 36 de 2012) y Universidad del Valle (2013: Acuerdo 004 de 2013). 
La Política institucional para la inclusión educativa de las personas con discapacidad en la Universidad Nacional de Colombia se focaliza en la población estudiantil en relación con la generación de condiciones de accesibilidad arquitectónica y simbólica; la disposición de ajustes de orden académico curricular; la formación de docentes y de la comunidad universitaria; y el trabajo con las empresas y la promoción de la producción académica en torno al tema (Universidad Nacional de Colombia 2012).

\section{Reconocimiento de las personas con discapacidad en la Universidad del Valle}

En correspondencia con el papel que desempeñan las instituciones de educación superior en la formulación de políticas sociales (Unesco e IESAL 2006), la Universidad del Valle hizo presencia directa en el diseño de la política de discapacidad tanto nacional, desde el constructo de las regiones (CPPS y CAF 2002), como de la municipal (Otoya, Quintana y Tenorio 2003). Desde la comprensión de la relación existente entre los factores ambientales que configuran el entorno de una persona con discapacidad, su desempeño y participación social (OMS 2001), el diseño de políticas se justifica. Esta relación orienta intervenciones con el fin de generar o fortalecer las condiciones del contexto para que esta población experimente menos limitaciones en el desempeño de sus actividades y menos restricciones en la participación social. La presencia de políticas en materia de discapacidad actúa como facilitadora para dicho propósito y es uno de los factores ambientales que propone la CIF (OMS 2001). Por ello, para el contexto de la Universidad del Valle, se hizo pertinente la construcción de la política como expresión de una postura institucional frente a un asunto y una población minoritaria en riesgo de experimentar situaciones de desigualdad. La discapacidad como un asunto complejo de orden multidimensional y multifactorial requiere, para su intervención desde el entorno universitario, la instalación y puesta en marcha de una política que involucre a los diferentes actores que constituyen la comunidad, así como instancias y procesos que le den soporte a la estructura y funcionamiento institucional. Para ello, su formulación debe constituirse desde las voces de las personas integrantes de dicha comunidad con quienes el debate y la puesta en común posibilite no solo la toma de decisiones, sino además la deconstrucción de imaginarios que marcan formas de relación excluyentes.

La Política de discapacidad e inclusión de la Universidad del Valle plantea una perspectiva que pone su énfasis en los dos fenómenos sociales que se viven en relación con la población con discapacidad en el contexto universitario: la discapacidad y la inclusión; estos conceptos requieren ser comprendidos y abordados estructuralmente de modo que garanticen el libre desarrollo y participación de estas personas en la cotidianidad de la universidad. La discapacidad no como condición biológica inherente a las personas. En su lugar, la discapacidad como un fenómeno multifactorial que se configura culturalmente en la interacción de los sujetos que tienen un funcionamiento corporal diverso con los entornos cuyas características favorecen o no la participación y el desarrollo. En el caso de la inclusión, entendida como un fenómeno opuesto a la exclusión que traducido como principio de actuación 
asume que todos hacemos parte de la comunidad universitaria como un asunto de derechos y de reconocimiento a las capacidades de aprendizaje, de desempeño diverso y de aporte para construir sociedad. Implica, por tanto, la responsabilidad individual e institucional de crear y mantener condiciones para asegurar la oportunidad de participar y decidir con plena autonomía, relacionarse y disfrutar de un bien, servicio o ambiente, junto con los demás ciudadanos, sin ninguna limitación o restricción por motivo de discapacidad (Universidad del Valle 2013, Num. 3, Art. 2).

Dicha política reconoce la presencia de sujetos diversos en su funcionamiento humano que hacen parte de la comunidad universitaria y que al asumir roles propios de la cultura institucional (profesor, estudiante, profesional, técnico, bibliotecólogo, secretario, contador, amigo, compañero, entre tantos otros roles) pueden enfrentarse a situaciones que vulneren el goce de sus libertades al no lograr involucrarse e interactuar autónomamente. Por tanto, en primera instancia estos dos asuntos, la discapacidad y la inclusión, requieren ser comprendidos y abordados desde las funciones sustantivas de la Universidad; esto significa la formación de talento humano en y desde la diversidad, la investigación y la extensión, como medios que posibiliten cumplir a la Universidad con su misión de forjar respuestas sociales para las situaciones de inequidad que vive dicha población, en coherencia con el lugar otorgado desde la década de 1990 al plantear el principio de educación para todos (Unesco y Ministerio de Educación y Ciencia de España 1994). Llegar al planteamiento de esta política en la Universidad puede ser evidencia de un cambio paradigmático en la conceptualización de la discapacidad por parte de la comunidad universitaria; no obstante, será necesario hacer el rastreo de su implementación para identificar su impacto en la configuración de nuevas realidades.

El posicionamiento de las personas con discapacidad es un elemento a resaltar en este cambio al pasar de ser objeto a ser sujeto en la definición de las condiciones requeridas para el disfrute de sus libertades. Esto le demanda una mirada sobre sí mismo y, más aún, trascender de la anécdota individual a la voz colectiva reflexiva, deliberativa y representativa para la construcción de otras realidades. El sujeto del que se habla en este contexto de política universitaria trasciende del que ha sido directamente beneficiario de los desarrollos en el ámbito de la educación superior inclusiva. El sujeto con discapacidad del que habla la universidad inclusiva generalmente es el estudiante a quien se dirigen todas las acciones con el fin de garantizar condiciones de equidad para su ingreso, permanencia y titulación. No obstante, la universidad inclusiva debe pensarse desde un sentido de comunidad universitaria en la que habitan e interactúan sujetos con diferentes condiciones de discapacidad, de diferentes procedencias étnicas, geográficas, familiares, socio-económicas y culturales que asumen múltiples roles: ser estudiante, docente, secretario(a), admnistrador(a), aseador(a), bibliotecario(a), entre muchos. Estos sujetos cohesionados desde una identidad que se construye colectivamente demandan una respuesta institucional que responda a la dialéctica entre la igualdad basada en las libertades para todos y la diferencia que surge ante el imperativo de reconocer las desigualdades en este contexto que pueden menoscabar el sentimiento de dignidad (Rawls 1971 en Moreno 2011). Esto implica, entre otros sentidos, reconocer los diversos modos de realización de la vida en la universidad que no necesariamente son los 
típicamente reconocidos por la comunidad mayoritaria y que requieren de diversas opciones, rutas, maneras, escenarios (Nussbaum 2006).

\section{Características del proceso de construcción de la política de discapacidad e inclusión en la Universidad del Valle}

La identificación de características que marcaron el proceso de construcción de la Política de discapacidad e inclusión de la Universidad del Valle se dio desde la comprensión de la sistematización como camino investigativo para objetivar, reflexionar y aprender de la experiencia (Jara 1992; Carvajal 2010). Se asume el ejercicio investigativo como un proceso interpretativo para reconocer lo actuado como proceso vivido y poder revalorarlo para recoger aprendizajes críticos que la misma experiencia proporciona. La sistematización implicó comprender más allá del producto el proceso mismo, en el contexto de un momento histórico en el que las personas con discapacidad asumen la voz sobre su futuro y se definen formas de interlocución conjunta con diferentes actores de la comunidad universitaria para resolver un asunto que es competencia de todos: la constitución de un entorno universitario que reconoce y responde a la presencia de las personas con discapacidad.

El ejercicio de sistematización se realizó en el marco de la práctica formativa de estudiantes de último año de fonoaudiología y terapia ocupacional de la Escuela de Rehabilitación Humana de la Universidad del Valle donde se precisó la conjugación de saberes desde estas dos disciplinas con otras como trabajo social, sociología, pedagogía y educación, en una intención de avanzar en procesos de intervención transdisciplinar sobre los ambientes generadores de discapacidad en un contexto tan importante para la realización de las personas con discapacidad como lo es el ámbito universitario.

En el proceso de construcción de la política de discapacidad se contó con la participación de personas con diferentes condiciones de discapacidad, así como sin discapacidad cuyo rol en el contexto universitario era de estudiante, profesor, funcionario no docente, egresado o jubilado.

Para la identificación de las características del proceso se tomaron como base los documentos construidos en la primera etapa a cargo del denominado "Grupo Gestor" (2007), así como las actas, relatorías, informes y productos del proceso en la segunda etapa comprendida entre 2009 y 2013 a cargo del grupo de profesoras y estudiantes del Programa "Rediversia" en el marco de la asignatura de "práctica profesional en fonoaudiología I y II" (Universidad del Valle 2010; 2011; 2012). Otro insumo utilizado fue el estudio de Hurtado, Jaramillo y Núñez (2013), en el cual se identificaron actores relevantes y acciones desarrolladas en la Universidad del Valle en el período 1992-2012 para responder a la discapacidad en el marco de la educación superior inclusiva.

Por tanto, esta primera mirada sobre el proceso vivido para la adopción de la política institucional de discapacidad (Universidad del Valle 2013) tomó como referente el período 2003-2012 al corresponder con la movilización de la comunidad universitaria en torno al asunto que desembocó en dicha construcción. 
De la revisión y análisis del proceso reconstruido, emergieron tres categorías bajo las cuales se hace una primera mirada:

1. Carácter participativo en la construcción de política con principio de corresponsabilidad.

2. Sentido formativo y de construcción en espiral.

3. Estrategia de trabajo en red.

\subsection{Carácter participativo y de corresponsabilidad en la construcción de la política}

La riqueza de la vida humana en el espacio universitario es uno de los propósitos de plantearse una política que brinde mayores condiciones de participación con autonomía. Como lo plantea Sen (2004 en Moreno 2011), se conceptúa la vida humana como un conjunto de seres y haceres que configuran las realizaciones o funcionamientos. La educación y el trabajo son en este caso determinantes directos de la capacidad humana. Por tanto, al ser la persona el centro de las preocupaciones con el fin de lograr definir las condiciones para ejercer con libertad las diferentes formas de hacer o ser, la presencia dinámica de los involucrados se hace indispensable con el fin de preservar las libertades para elegir de una amplia gama de posibilidades aquellas que más se ajustan a la realidad de cada sujeto.

Por tanto, la presencia de diversos actores en el proceso de construcción de la política es una de las formas de agenciar el modo de vida que se quiere o requiere en el escenario universitario. Los integrantes de la comunidad universitaria tuvieron la libertad de hacer parte del proceso en los tiempos y formas que cada uno planteara. En este sentido se puso en juego un principio liberal democrático (Rawls 1971 en Moreno 2011) para que toda persona tuviera la misma posibilidad u ocasión (oportunidad), en el tiempo y en el espacio, de proponer la universidad inclusiva que se requería conforme a sus intereses y capacidades.

Por otra parte, la participación es un asunto esencial en el marco de la democracia, y en la medida que la comunidad es más activa y organizada se potenciaba la creación de espacios de interlocución y de negociación (Ziiccardi 2004), hecho que lleva a resaltar la dinámica participativa como pieza clave para la construcción de consensos a partir de los intereses y necesidades individuales, así como de la presencia de disensos. En este proceso se evidenció, precisamente, la movilización de todos aquellos que se sintieron implicados o identificados con la discapacidad y con la construcción de un entorno universitario que expresa situaciones de desigualdad en la cotidianidad de la vida universitaria.

Este fue un proceso en el cual la voz de cada uno, desde su experiencia con la discapacidad, permitió concretar un sentir y una demanda común en relación con el posicionamiento institucional frente a la discapacidad y a las personas con discapacidad en esta Universidad. Por tanto, el carácter participativo se evidenció a través de mecanismos de deliberación (Ziiccardi 2004) desde la concepción y construcción hasta la adopción de la política; y se hizo más potente y sostenido al surgir de grupos de base organizados que venían sintiéndose insatisfechos frente a la realidad de la población con discapacidad en la Universidad. 
También el proceso participativo tuvo una relación con lo identitario que logró constituirse desde un sentido común en torno a la vida en la universidad dejando de lado la tradicional relación de poder entre el profesional de la salud que define la identidad del otro. Esto desde el cuestionamiento que hace el modelo social a que la identidad de la persona con discapacidad se tenga que construir en función de términos médicos relativos a las deficiencias personales como físicas, sensoriales o intelectuales (Barton 1998). En este caso de la construcción de la política, el elemento de cohesión lo constituyó la búsqueda de alternativas institucionales para generar condiciones de igualdad en los derechos para acceder y disfrutar de la vida universitaria en las condiciones requeridas por cada uno de los miembros de la comunidad universitaria.

Ser consecuentes con una perspectiva de derechos en la constitución de entornos universitarios inclusivos convoca al permanente "reconocimiento" de las personas con discapacidad como sujetos de derechos y de responsabilidades. Esto implica una actitud corresponsable frente a la problematización y al planteamiento de respuestas en la medida que se tenga presente que el derecho propio no debe trasgredir el derecho del otro. La acción conjunta, dialogada y deliberada entre unos y otros ha de sostenerse en el tiempo para la constitución de espacios universitarios en los que sea posible habitar, interactuar e involucrarse con autonomía y libertad sin vulnerar el derecho de ningún integrante de la comunidad universitaria. Por tanto, el diseño participativo de una política como la de discapacidad e inclusión de la Universidad del Valle se presentó como oportunidad para configurar nuevas formas de comprender la institución, la vida universitaria y al "otro tan diferente como yo".

El sentido de corresponsabilidad se fue armando a medida que se avanzó en la comprensión de la complejidad de la realidad que se abordó, y propició la implicación de los actores en la búsqueda de alternativas frente a situaciones colectivas y no frente a las individuales, al asumir que se es sujeto de derechos e igualmente sujeto de responsabilidad social. La discapacidad comprendida como un asunto multidimensional y multifactorial demanda el compromiso de diferentes actores para comprender y aportar en la definición de alternativas en el contexto de la vida universitaria. Se resalta el concurso de personas con discapacidad que asumen diversos roles: estudiantes, funcionarios docentes, funcionarios no docentes, egresados o aspirantes a ingresar a la Universidad; igualmente, hicieron parte del proceso monitores de los estudiantes con discapacidad, profesores que hubieran tenido relación pedagógica con estudiantes con discapacidad, jubilados de la Universidad, directivos universitarios y grupos organizados de la comunidad universitaria: Colectivo de Discapacidad, Colectivo Sordo y Colectivo RBC (tabla l).

La movilización y los procesos organizativos sostenidos por parte de la comunidad universitaria con y sin discapacidad dieron cuenta del valor de la cohesión para lograr incidir en la estructura universitaria. Esta cohesión estuvo marcada por la identidad frente a la situación. Por otra parte, la constitución de diferentes formas organizativas de los actores participantes a lo largo del período analizado evidenció una perspectiva para afrontar la discapacidad, posiblemente permeada por los elementos sociales, culturales y políticos de la década de 1960, en la cual se reconoció la discapacidad como fenómeno social, y no solo biológico. 
Tabla 1. Grupos organizados de la comunidad universitaria que participaron del proceso de construcción de la política de discapacidad e inclusión

\begin{tabular}{|l|l|l|}
\hline \multicolumn{1}{|c|}{ Año } & \multicolumn{1}{|c|}{ Grupo organizado } & \multicolumn{1}{c|}{ Integrantes } \\
\hline 2003 & Sindicato Sintraunicol & Funcionarios públicos no docentes \\
\hline 2003 & $\begin{array}{l}\text { Comité de atención a la } \\
\text { discapacidad }\end{array}$ & $\begin{array}{l}\text { - Director salud ocupacional } \\
\text { - Representante de la Escuela de Rehabilitación } \\
\text { Humana de la Facultad de Salud (ERH) } \\
\text { - Vicerrectoría Académica }\end{array}$ \\
\hline 2007 & Gepresentante trabajadores \\
\hline $2009-2010$ & Colectivo Sordo & $\begin{array}{l}\text { - Estudiantes con y sin discapacidad } \\
\text { - Profesores } \\
\text { - Comité de discapacidad }\end{array}$ \\
\hline $2011-2012$ & $\begin{array}{l}\text { - Aspirantes sordos a ingresar a la educación superior } \\
\text { - Estudiantes oyentes de diversos programas académi- } \\
\text { cos (fonoaudiología, trabajo social, psicología) } \\
\text { - Profesores }\end{array}$ \\
\hline $2009-2013$ & Rediversia & $\begin{array}{l}\text { Estudiantes con y sin discapacidad de la Escuela de } \\
\text { Rehabilitación Humana }\end{array}$ \\
\hline
\end{tabular}

Fuente: elaboración propia.

En los inicios del proceso de hacer visible en el entorno universitario la población con discapacidad, la movilización sindical dio un paso adelante con la instalación del primer Comité de atención a los discapacitados (Universidad del Valle 2003; 2010; Hurtado, Jaramillo y Núñez 2013). Los grupos y colectivos que se fueron configurando a lo largo del proceso de construcción de la política estuvieron constituidos por diversos actores de la comunidad universitaria interesados en aportar desde una mirada reconocedora de los derechos de las personas con discapacidad. Estos fueron el Grupo Gestor, el Colectivo de Discapacidad, el Colectivo RBC y Rediversia.

Producto del proceso deliberativo, se construyeron seis compromisos que se desarrollan a través de 17 estrategias (Universidad del Valle 2013), bajo las siguientes consideraciones:

\subsubsection{Construcción de una cultura institucional de reconocimiento de la discapacidad}

Reconocer la discapacidad implica trascender en la forma de relacionarse con dicho fenómeno social y con las personas con discapacidad. Revisar las concepciones que se tienen y movilizar nuevas formas de comprensión pueden ser posibles a través de diversos mecanismos que permitan informarse, interactuar con dicha realidad para reconstituir el concepto que se 
tiene de "ese otro" cuya diferencia es valorada desde el déficit. Para ello, se hace necesario abrir espacios permanentes de interacción con dicha realidad y de reflexión que propicie la reconstrucción de percepciones y de imaginarios y que involucre a los diferentes actores de la comunidad universitaria. Particularmente el grupo docente debe contar con dichos procesos formativos que le lleven a reconfigurar al sujeto universitario que aprende con el fin de que este pueda transitar a la dimensión pedagógica y didáctica con una actitud creativa y reconocedora de las diversas formas de aprender y de enseñar.

Para el grupo de participantes de este proceso, la acción formativa no se dirigió solo al docente; se pensó en la comunidad universitaria en general desde el reconocimiento que la vida universitaria acontece en múltiples espacios cotidianos más allá del salón de clase desde los cuales se propician condiciones de desarrollo. Por ello, fue pertinente involucrar en estos procesos formativos a la secretaria, el vigilante, el bibliotecario, el directivo, el decano, entre otros.

Los participantes del proceso consideraron que la proyección de la vida laboral del profesional con discapacidad implica establecer puentes entre la Universidad y el sector empresarial que son indispensables con el fin de que los empleadores reconfiguren la imagen que tienen de profesional con discapacidad y se aporte en la creación de condiciones para la vinculación laboral y el desarrollo profesional.

Por último, los integrantes de este proceso de construcción pensaron la formación universitaria "en y para la diversidad", hecho que convoca a la revisión de los currículos para proyectar el enriquecimiento en la formación integral del profesional de la Universidad del Valle. Todo profesional debe formarse reconociendo las diferencias y valorándolas, así como con la capacidad de identificar las inequidades sociales, a las cuales la sociedad espera que aporte desde su rol profesional.

\subsubsection{Información y divulgación disponible y accesible sobre discapacidad}

La toma de decisiones fundamentadas para reducir las inequidades en el entorno universitario es posible si se cuenta con información fiable y permanente sobre las situaciones que viven las personas con discapacidad en la cotidianidad de la universidad. Por tanto, los participantes del proceso consideraron la necesidad de trabajar por la constitución de un mecanismo institucional que asegure disponer de dicha información como soporte para comprender la realidad de los integrantes de la comunidad universitaria con discapacidad y responder adecuadamente para reducir la inequidad desde el marco del reconocimiento a los derechos.

Por otra parte, los participantes del proceso plantearon la relevancia de generar mecanismos de información y divulgación sobre la política, la discapacidad, las personas con discapacidad y la comunidad universitaria considerando que esta realidad debe hacerse más visible en el contexto universitario. 


\subsubsection{Acceso, permanencia y preparación para el egreso de los miembros de su comunidad universitaria}

Los participantes direccionaron este compromiso hacia tres grupos poblacionales con discapacidad específicos: estudiantes, funcionarios docentes y funcionarios no docentes, quienes viven expuestos de manera permanente a la vulneración de sus derechos. Desde la convicción de la capacidad de desempeño y desarrollo que tiene cada persona con discapacidad, así como el reconocimiento de la ruptura que se presenta en su participación autónoma por las características de los entornos que han sido diseñados bajo una concepción de "normalidad", se planteó la necesidad de avanzar hacia el diseño de programas de acompañamiento que informen, orienten y gestionen condiciones de equidad en el proceso de ingreso, durante los años de permanencia en la institución, así como en su proceso de egreso de esta etapa de la vida. Este compromiso, en relación con los aspirantes y estudiantes, implica tocar la didáctica, el modelo pedagógico, las formas de evaluación, así como las mediaciones que se pueden plantear para favorecer el desarrollo académico de los estudiantes con discapacidad. Las condiciones de ingreso son un asunto en discusión permanente, frente a las actuales políticas de excepción para otros grupos en desventaja ya que para discapacidad no existen cupos de excepción. En el caso de los profesores y funcionarios no docentes con discapacidad, implica una actuación concertada desde el servicio médico y salud ocupacional que brinde soporte para un pleno desempeño laboral acorde con su cargo y perfil ocupacional.

\subsubsection{Accesibilidad arquitectónica y simbólica}

El acceso libre y autónomo a los diferentes espacios arquitectónicos y sociales es una necesidad de base para participar en la vida universitaria. Desde allí, los participantes del proceso plantearon el compromiso que tiene la Universidad por hacer cada vez más accesible los diferentes espacios públicos, así como los diversos medios de información y comunicación. Para ello, pensar el tema de diseño universal es un principio de soporte, sin negar la importancia de propiciar el uso de tecnología de asistencia para hacer adaptaciones específicas que son requeridas por algunas personas con discapacidad. Este compromiso convoca a pensar particularmente en los funcionamientos tan diversos que tiene el ser humano que le llevan a hacer todo lo que hace cualquier sujeto pero de manera diferente: desplazarse con la ayuda de un dispositivo, escribir con la ayuda de un punzón o de un apuntador, leer con apoyo auditivo o en braille; comunicarse a través de otra lengua, presentar evaluaciones en formatos orales, entre otros.

\subsubsection{Fortalecimiento de la participación en torno al tema}

La población con discapacidad debe participar de las discusiones y toma de decisiones sobre situaciones que les competen directamente para forjar su futuro y las mejores formas de realización (Rawls 1971 en Moreno 2011). Este es un sentir y una demanda explícita de los integrantes del proceso de construcción de la política. La voz de cada integrante de la comunidad universitaria tiene un 
lugar desde su reconocimiento como sujeto de derechos y de deberes; ante todo requieren ser reconocidos y valorados como sujetos activos en los procesos y no como objetos de intervención. Para ello, es importante abrir y mantener espacios que construyan actitud política en relación con la discapacidad, la inclusión y la exclusión en la universidad como un asunto a ser abordado con otros, es decir, con la comunidad universitaria, otras universidades, entes territoriales y sociedad civil organizada.

\subsubsection{Fortalecimiento de la investigación en discapacidad}

Reconociendo el valor de la investigación para aportar en la comprensión de realidades, en la toma de decisiones, así como en la valoración de procesos de intervención, los participantes de este proceso plantearon la importancia de otorgar un lugar visible a la investigación en discapacidad toda vez que la Universidad tiene un compromiso social para enfrentar las desigualdades sociales en la región, así como en la formación de futuros investigadores. El fortalecimiento de la capacidad investigativa en discapacidad, de la mano de su direccionamiento desde las necesidades que se definan en la región y el país es una responsabilidad de la academia para hacer visible el aporte de la investigación en este campo.

En la tabla 2 se relacionan los compromisos enunciados con las propuestas de estrategias, las cuales son la base para la puesta en marcha de programas, proyectos, servicios o acciones específicas.

En este proceso participativo con un énfasis deliberativo, otra característica que se puso en evidencia fue el sentido de abajo hacia arriba en la construcción de condiciones que fueron ganando progresivamente una representatividad democrática a través de la constitución de una instancia institucional desde la cual se estudia, analiza, define, recomienda y coordina la realización de las acciones para propiciar los cambios requeridos. Se refiere a la constitución de un Comité Institucional con representación de la población con discapacidad y directivas universitarias. La instalación del Comité Institucional de Discapacidad como expresión del mecanismo participativo propuesto generó dos niveles de responsabilidad. En primera instancia, otorga una responsabilidad a la población con discapacidad de la institución en la medida en que de ellos depende la decisión de elegir democráticamente quién los represente. Por otra parte, aquellas personas que representan un colectivo cuentan con la responsabilidad de hacer presencia activa en la deliberación para el diseño, implementación y monitoreo de las acciones en torno a los compromisos asumidos por la Universidad para su implementación desde diversas instancias.

La presencia de esta instancia de representación democrática implicó un paso para modificar la tendencia de respuestas instaladas exclusivamente desde la instancia de "Bienestar Institucional" como un asunto centrado en las personas con discapacidad hacia un abordaje que compromete a otras instancia desde las implicaciones académico-curriculares, de investigación, proyección a la comunidad, así como de infraestructura física y arquitectónica y de sistema de información y divulgación, entre otros. 
Tabla 2. Compromisos asumidos institucionalmente para responder a la discapacidad e inclusión en la Universidad del Valle

\begin{tabular}{|c|c|}
\hline Asunto de la política & Estrategia \\
\hline $\begin{array}{l}\text { Construcción de una cultura } \\
\text { institucional de reconocimiento } \\
\text { de la discapacidad }\end{array}$ & $\begin{array}{l}\text { - Toma de conciencia en la relación Universidad - empresa } \\
\text { para el fortalecimiento de los procesos de vinculación } \\
\text { laboral de los egresados con discapacidad. } \\
\text { - Cualificación docente para la construcción de respuestas } \\
\text { diferenciales acordes con las particularidades de los } \\
\text { estudiantes con discapacidad. } \\
\text { - Formación integral de los profesionales de la Universidad } \\
\text { del Valle reconocedores de la diversidad y promotores de } \\
\text { condiciones de equidad bajo el reconocimiento de los } \\
\text { derechos de las personas con discapacidad. }\end{array}$ \\
\hline $\begin{array}{l}\text { Información y divulgación } \\
\text { disponible y accesible sobre } \\
\text { discapacidad en la Universidad }\end{array}$ & $\begin{array}{l}\text { - Disposición de información sobre la población con } \\
\text { discapacidad en la Universidad. } \\
\text { - Comunicación sobre acciones de política a través de los } \\
\text { sistemas y medios de comunicación que maneja la } \\
\text { institución. }\end{array}$ \\
\hline $\begin{array}{l}\text { Acceso, permanencia y } \\
\text { preparación para el egreso de los } \\
\text { miembros de su comunidad } \\
\text { universitaria }\end{array}$ & $\begin{array}{l}\text { - Programa de acompañamiento al estudiante con } \\
\text { discapacidad. } \\
\text { - Programa de acompañamiento al funcionario. } \\
\text { - Innovación y virtualización pedagógica para el acceso } \\
\text { democrático al conocimiento. }\end{array}$ \\
\hline $\begin{array}{l}\text { Accesibilidad arquitectónica y } \\
\text { simbólica }\end{array}$ & $\begin{array}{l}\text { - Accesibilidad de los sistemas y mecanismos de información } \\
\text { y divulgación que aneja la Universidad del Valle. } \\
\text { - Adopción normativa de accesibilidad y principios de diseño } \\
\text { universal. } \\
\text { - Disposición de recursos tecnológicos para mayor autonomía } \\
\text { de las personas con discapacidad en la vida universitaria. }\end{array}$ \\
\hline $\begin{array}{l}\text { Fortalecimiento de la } \\
\text { participación en torno al tema }\end{array}$ & $\begin{array}{l}\text { Fortalecimiento de procesos organizativos y participativos } \\
\text { en torno a la discusión y toma de decisiones sobre } \\
\text { discapacidad. }\end{array}$ \\
\hline $\begin{array}{l}\text { Fortalecimiento de la } \\
\text { investigación en discapacidad }\end{array}$ & $\begin{array}{l}\text { - Impulso de investigación, transferencia de conocimiento } \\
\text { para la toma de decisiones sobre discapacidad y } \\
\text { planteamiento de alternativas de atención. }\end{array}$ \\
\hline
\end{tabular}

Fuente: elaboración propia a partir de Universidad del Valle (2013).

Mantener el sentido ascendente implicó un ejercicio político para lograr la articulación con los procesos y estructura de la Universidad. De esta manera, la propuesta fue constituyéndose en un valor compartido que logró permear las instancias de toma de decisión institucional en las cuales se aprobó y adoptó una postura frente al fenómeno de la discapacidad en la Universidad.

Finalmente, este proceso participativo marcado por la construcción de lo individual a lo grupal y de ahí a lo institucional trascendió para instalarse en un nivel de participación interinstitucional a través de la interlocución en espacios de ciudad, país y región para conversar, comprender y valorar otras formas de hacer para volver hacia adentro. Esto se evidenció en tres espacios que convocan al trabajo en red regional, nacional e internacional ${ }^{2}$.

2 Estos espacios de participación interinstitucional se refieren a la "Mesa Interuniversitaria por la Discapacidad", la "Red Colombiana de Instituciones de Educación Superior por la Discapacidad” y la "Red Interuniversitaria Latinoamericana y del Caribe sobre la discapacidad y los Derechos 
En consecuencia este camino, permeado por la emergencia de nuevos paradigmas que implican la noción de discapacidad y de sujeto coherente con un modelo de participación para el ejercicio de la ciudadanía y para la constitución de sociedad (Díaz 2009), contó con el valor de la participación social desde el sentido de "ser más sujeto y menos objeto de las decisiones que les conciernen a los ciudadanos" (Hopenhayn 1988), al ser parte de un ejercicio de deliberación entre actores universitarios con y sin discapacidad en función de una propuesta que llevó a entender lo institucional como parte constitutiva de lo colectivo y no como opositora del bien común.

La participación se caracterizó por ser una acción intencionada y activa de los sujetos y grupos presentes en el contexto donde se tejieron tramas concretas de relaciones sociales y de relaciones de poder (Velásquez 2008), que generaron coaliciones y tensiones resueltas en la medida que se posibilitó la interlocución horizontal para la discusión y el consenso de aquello que se debía mantener, transformar o eliminar de la realidad institucional para avanzar hacia la constitución de condiciones requeridas.

No obstante, este sentido de participación no se evidenció plenamente. No se logró una movilización masiva frente al tema, situación que pone en cuestión la manera de asumirse como sujeto político con la capacidad de tomar decisiones relacionadas con los ámbitos que rigen su vida. Es posible que esto se haya dado por razones centradas en no sentirse implicado con dicha realidad al no vivirla directamente o en un sentido identitario, en el cual una persona con algún tipo de condición de salud no se asuma como persona con discapacidad y, por tanto, no se sienta convocado:

\footnotetext{
El perfecto ciudadano informado, activo y coherente ha sido una expresión muy minoritaria en las sociedades occidentales, porque a pesar del incremento del nivel educativo y un mayor acceso a la información, el tiempo, las energías y la intensidad participativa son recursos limitados y ejercidos de manera muy desigual por los ciudadanos (Ziiccardi 2004, 12).
}

\subsection{Sentido formativo y de construcción en espiral}

Este proceso desarrollado en el período 2003-2012 se define con dos sentidos fundamentales que explican el tiempo que tomó la adopción de la política: formación y construcción permanente; desde el criterio de tiempo podría plantearse como un proceso ineficiente. No obstante, es claro que toma tiempo la instalación de un tema que no es visible y que, aparentemente, es relevante en el contexto académico solo para aquellos que lo abordan como objeto de estudio. Por tanto, fue importante permear sujetos e instancias sostenidamente para comprometer voluntades y al tiempo movilizar las formas de comprender la discapacidad; esto como requisito necesario para proponer respuestas asertivas en un espacio de trascendencia social como lo es la Universidad. La interacción con personas con discapacidad y la articulación del proceso a la información

Humanos". En estos espacios de participación se trabaja colaborativamente para construir referentes comunes, nutrirse mutuamente de los procesos y desarrollos de cada institución, región y país, así como para desarrollar agendas comunes en función del propósito de fortalecer los espacios inclusivos en sus instituciones. 
sobre el tema fueron dos elementos formativos de importancia (Universidad del Valle 2011; 2012; 2013) marcados por la discusión, reflexión y construcción colectiva, donde se evidenció la presencia y posicionamiento de las directivas universitarias frente al tema.

La Declaración sobre los Derechos de las Personas con Discapacidad (Unesco e IESALC 2006) tuvo un lugar privilegiado en la construcción, junto con el marco constitucional y legal existente en el país al definir condiciones de equidad. Por tanto, estos marcos dialogaron con el Proyecto educativo institucional (Universidad del Valle 2002) en búsqueda de su armonización.

De otra parte, el proceso logró una construcción conceptual en espiral y en una dirección ascendente. Esto se evidenció en el enriquecimiento progresivo y permanente, en la comprensión de la realidad institucional desde un marco conceptual de la discapacidad que se distanciara de la perspectiva del déficit y se nutriera del modelo social. Este sentido de construcción conceptual en espiral implicó un ejercicio progresivo que partió de la socialización de las experiencias y anécdotas individuales hacia la identificación de sentidos comunes que fueran generando una sintonización entre los actores participantes. Con este ambiente construido, fue posible trascender a la discriminación de la información, su organización y estructuración para llegar a un nivel de formalización con una identidad colectiva a partir de los principios compartidos para asumir institucionalmente una postura frente a la realidad de las personas con discapacidad.

\subsection{Trabajo en red para lograr mayor incidencia en la Universidad}

En Colombia, para la formulación de políticas públicas en discapacidad se han constituido procesos consultivos y participativos que vinculan organizaciones e individuos que representan instituciones no gubernamentales y de la sociedad civil para poner en marcha principios de participación, autonomía y no discriminación emanados de diferentes instrumentos internacionales (Otoya, Quintana y Tenorio 2003). La acción articulada de estas diferentes instancias se hace indispensable en la medida que se comprende que el asunto de la discapacidad es complejo en su configuración y abordaje, por cuanto requiere la actuación concertada de diferentes sectores.

De igual forma, esta mirada ha de mantenerse dentro de un espacio como el universitario. La Universidad del Valle cuenta con una estructura de alta complejidad para responder a las necesidades de sus unidades académicas, instancias y estamentos. En este contexto, la comunidad universitaria está conformada por estudiantes, egresados, docentes y funcionarios no docentes que convergen en espacios de interacción donde se construye la huella institucional. La organización de grupos de base fue en este proceso, y seguirá siendo, uno de los mecanismos que permitieron construir identidad al involucrarse con asuntos esenciales de la vida universitaria.

Por tanto, un sentido de trabajo en red que se planteó fue el de la articulación de los diferentes actores e instancias para enfrentar problemas que no podían ser siquiera apreciados adecuadamente desde cada sujeto o instancia en forma aislada. Este ejercicio colectivo que fue construyendo identidad en el intercambio 
entre los diferentes actores aportó en la formación política desde la convicción del poder de la fuerza colectiva para propiciar cambios de una realidad. El intercambio a través del diálogo argumentado se dio en el marco de la libertad, de forma que la opinión, la propuesta y la contrapropuesta, así como el ingreso y la permanencia en el proceso no tuvieron ningún tipo de restricción. Además, esta interlocución se dio a partir del establecimiento de relaciones horizontales más allá del cargo e instancia institucional representada por cada actor.

Un trabajo en red se sostiene no solo por la presencia de los sujetos. Tiene mucho más peso la relación que se teje con el otro y los otros desde un espíritu colaborativo y un sentido de libertad para hacer parte y para construir opinión pública, propositiva e informada donde el valor común, en este caso "vida universitaria y garantía de derechos", fue el elemento articulador. Será necesario sostener la deliberación y construcción de consenso, así como el fortalecimiento de las sinergias para brindar soporte a los cambios sustanciales que plantea la política.

\section{Conclusiones}

Como lo plantea Franco y Álvarez (2008), una política pública tiene sentido si se apoya en los derechos humanos mediante mecanismos de construcción ciudadana y participación política. Al tiempo, un proceso participativo que avance hacia el paradigma de la autonomía (Díaz 2009) afianza el ejercicio de los derechos desde un fin primordial: modificar el entorno social. Por tanto, la discapacidad comprendida desde una perspectiva relacional e interactiva del sujeto con su entorno seguirá demandando acciones, en este caso, hacia el entorno universitario, de manera que se continúen eliminando las barreras y obstáculos sociales que se manifiestan, particularmente, en las representaciones simbólicas de la sociedad (Sen 2004 en Moreno 2011); estas son la base de muchas otras barreras que se eliminan desde las decisiones y acciones humanas.

Si bien la política institucional es soporte, es necesario advertir que no es suficiente. Es soporte porque visibiliza la realidad de una población, sienta la postura institucional y define unas apuestas como universidad reconocedora de las diferencias. Sin embargo, no es suficiente para avanzar en la reconstitución de espacios y relaciones que garanticen un cambio en la realidad de las personas con discapacidad si no va acompañado, de manera sostenida en el tiempo, de procesos formativos que contribuyan en la resignificación de la discapacidad y de las personas con discapacidad desde sus capacidades y aportes a la sociedad.

Es necesario sostener tres asuntos en este proceso permanente de resignificación: trascender de la concepción biológica, individual y medicalizada de la discapacidad para comprenderla como un proceso social complejo que supera a la persona; otorgar el lugar que le corresponde al entorno universitario como factor que aporta a la configuración de situaciones discapacitantes (desde sus características sociales, físicas, académicas, comunicativas, tecnológicas, laborales o arquitectónicas, entre otras) y, mantener los espacios de participación donde la voz de la persona con discapacidad no pierda su valor desde su presencia activa, reflexiva y deliberativa asumiéndose corresponsablemente como sujeto 
de deberes y de derechos, que no solo es beneficiario sino que aporta en la construcción de entornos sociales de reconocimiento mutuo desde la diferencia.

La mirada sobre el diseño de una política de discapacidad para un contexto educativo como el universitario, es un principio. Será necesario continuar con el proceso de identificar evidencias de su implementación y, especialmente, de los cambios experimentados por las personas con discapacidad en la cotidianidad de la vida en la universidad, como espacio para el desarrollo de sus capacidades y su realización humana. Esta será la base para la identificación de nuevos requerimientos que impulse acciones teniendo como principio de actuación el reconocimiento del valor de la diferencia para la construcción de entornos reconocedores de los derechos y de la dignidad humana.

\section{Referencias bibliográficas}

Alcantud, Francisco. «La inclusión de estudiantes con discapacidad en los estudios superiores: una reflexión después de diez años». En La "Declaración de Salamanca" sobre necesidades educativas especiales 10 años después: valoración y prospectiva, coordinado por Gerardo Echeita y Miguel Verdugo, 161-168. Salamanca: Universidad de Salamanca, Instituto Universitario de Integración en la Comunidad, 2004.

Barton, Len (comp.). Discapacidad y sociedad. $1^{\mathrm{a}}$ edición. España: Ediciones Morata, 1998.

Carvajal, Arizaldo. Teoría y práctica de la sistematización de experiencias. Cali: Facultad de Humanidades, Escuela de Trabajo Social y Desarrollo Humano, Universidad del Valle, 2010.

Díaz, Eduardo. «Reflexiones sociológicas para una sociología de la discapacidad». Revista sociológica de pensamiento crítico, Intersticios, Vol. 3, n 2, 2009: 85-99.

Ferreira, Miguel. «Aproximación sociológica a la discapacidad desde el modelo social. Apuntes caracteriológicos». Revista española de investigaciones sociológicas, $\mathrm{n}^{\circ} 124,2008:$ 141-174.

Franco, Álvaro y Carlos Álvarez. «Derechos humanos, una oportunidad para las políticas públicas en salud». En Revista políticas de salud y salud pública, Vol. 22, $\mathrm{n}^{\circ}$ 3, 2008: 280-286.

Grupo Gestor. Por una comunidad universitaria que re-conoce la diversidad. Informe final. Cali: Universidad del Valle, 2007.

Hopenhayn, Martín. «La participación y sus motivos». Chile: 1988. http:// es.scribd.com/doc/199735529/Hopenhayn-Martin-La-participacion-y-susmotivos\#scribd (último acceso: 28 de abril de 2015).

Hurtado, Camila, Ana Jaramillo y Vanessa Núñez. Reconocimiento de la discapacidad en la Universidad del Valle durante el período 1992-2012 en el marco de la educación superior inclusiva. Informe final de trabajo de grado. Cali: Escuela de Rehabilitación Humana, Programa Académico de Fonoaudiología, 2013.

Jara, Oscar. Para sistematizar experiencias. México D.F.: Alforja-IMDEC, 1992.

Jiménez, Antonio y Agustín Huete. «Políticas públicas sobre discapacidad en España. Hacia una perspectiva basada en los derechos». Política y Sociedad, Vol. 47, $\mathrm{n}^{\circ}$ 1, 2010: 137-152. 
Ministerio de Educación Nacional (MEN). Lineamientos de política de Educación Superior inclusiva. Bogotá: MEN, 2013.

Molina, Rocío. «Educación superior para estudiantes con discapacidad en Colombia». En XIV Congreso mundial de inclusión internacional 2006 Construir un futuro incluyente: un reto para la globalización. Confederación mexicana de organizaciones en favor de personas con discapacidad intelectual, A.C. (CONFE). Acapulco, 7 a 10 de noviembre de 2006. http://www.confe.org.mx/red/pdf4/ molina-educacion-superior.pdf (último acceso: 17 de octubre de 2014).

Moreno, Marisol. Infancia, políticas y discapacidad. Bogotá: Universidad Nacional de Colombia, 2011.

Nussbaum, Martha. Las fronteras de la justicia. Consideraciones sobre la exclusión social. Barcelona: Paidós, 2006.

Organisation for Economic Co-operation and Development (OECD). Sickness, Disability and Work: Breaking the Barriers. A Synthesis of Findings Across OECD Countries. París: OECD, 2010.

Organización de las Naciones Unidas (ONU). Declaración Universal de los Derechos Humanos. Nueva York: Asamblea General de las Naciones Unidas, 1948.

Organización de las Naciones Unidas (ONU). Convención sobre los derechos de las personas con discapacidad. Nueva York: Asamblea General de las Naciones Unidas, 2006.

Organización de las Naciones Unidas para la Educación, la Ciencia y la Cultura (Unesco) e Instituto Internacional para la Educación Superior en América Latina y el Caribe (IESALC). Informe sobre la Educación Superior en América Latina y el Caribe 2000-2005. La metamorfosis de la Educación Superior. Caracas: IESALC, 2006.

Organización de las Naciones Unidas para la Educación, la Ciencia y la Cultura (Unesco) y Ministerio de Educación y Ciencia de España. Declaración de Salamanca y Marco de Acción para las necesidades educativas especiales. Aprobado por la Conferencia mundial sobre necesidades educativas especiales: acceso y calidad. Madrid: Ministerio de Educación y Ciencia de España, 1994.

Organización de las Naciones Unidas para la Educación, la Ciencia y la Cultura (Unesco) y Ministerio de Educación y Ciencia de España. Informe de seguimiento de la EPT en el mundo. Llegar a los marginados. Resumen. París: Ediciones Unesco, 2010.

Organización Mundial de la Salud (OMS). Clasificación internacional del funcionamiento la discapacidad y la salud. Ginebra: OMS, 2001.

Organización Mundial de la Salud (OMS) y Banco Mundial (BM). Resumen del Informe mundial sobre la discapacidad. Malta: OMS, 2011.

Otoya, Cristina, Patricia Quintana y Liliana Tenorio. Política de atención a la discapacidad para el municipio de Santiago de Cali. Cali: Alcaldía de Santiago de Cali, Secretaria de Desarrollo Territorial y Bienestar Social, Escuela de Rehabilitación Humana, Universidad del Valle, 2003.

Palacios, Agustina y Francisco Bariffi. La discapacidad como una cuestión de derechos humanos. Una aproximación a la Convención Internacional sobre los Derechos de las Personas con Discapacidad. Madrid: Ediciones Cinca, 2007.

Pantano, Liliana. «Nuevas miradas en relación con la conceptualización de la discapacidad. Condición y situación de discapacidad». En Visiones y revisiones de 
la discapacidad, compilado por Patricia Brogna, 73-97. México D.F.: Fondo de Cultura Económica, 2009.

Parra, Carlos y María Palacios. «Enfoque de derechos en la política de discapacidad». Revista Civilizar, Ciencias Sociales y Humanas, Vol. 7, n² 13, 2007: 97-114.

Rama, Claudio. «La tercera reforma de la educación superior en América Latina y el Caribe». Buenos Aires: Fondo de Cultura Económica, 2006.

Rawls, John. Theory of Justice. Cambridge: Harvard University Press, 1971.

Roth, André-Noël. Políticas públicas. Formulación, implementación y evaluación. Bogotá: Ediciones Aurora, 2006.

Sassaki, Romeu. Inclusão: a universidade e a pessoa com deficiência. 2001. www. apacsp.com.br (último acceso: 26 de julio de 2003).

Sen, Amartya. «Discapacidad y justicia». Ponencia presentada en la Segunda conferencia internacional sobre discapacidad y desarrollo inclusivo. Washington: Banco Mundial, diciembre de 2004.

Universidad del Valle. Acuerdo ool del Consejo Superior mediante el cual se adopta el Proyecto educativo institucional de la Universidad del Valle. Cali: Universidad del Valle, 2002.

Universidad del Valle. Resolución 086 de Rectoría mediante la cual se constituye el Comité de Atención a los Discapacitados. Cali: Universidad del Valle, 2003.

Universidad del Valle. Rediversia. Proyecto de práctica fonoaudiológica 2010-2011. SERH. Cali: Programa Académico de Fonoaudiología. Escuela de Rehabilitación Humana, 2010.

Universidad del Valle. Rediversia. Proyecto de práctica fonoaudiológica 2011-2012. SERH. Cali: Programa Académico de Fonoaudiología. Escuela de Rehabilitación Humana, 2011.

Universidad del Valle. Rediversia. Proyecto de práctica fonoaudiológica 2012-2013. SERH. Cali: Programa Académico de Fonoaudiología. Escuela de Rehabilitación Humana, 2012.

Universidad del Valle. Acuerdo 004 del Consejo Superior mediante el cual se adopta la Política de discapacidad e inclusión de la Universidad del Valle. Cali: Universidad del Valle, 2013.

Universidad Nacional de Colombia. Acuerdo 036 de 2012 por el cual se establece la política institucional para la inclusión educativa de las personas con discapacidad en la Universidad Nacional de Colombia. Bogotá: Universidad Nacional de Colombia, 2012.

Velásquez, Elkin. «La gobernanza territorial en las ciudades: ¿De qué hablamos?». En Gobernabilidad territorial en las ciudades andinas. Organización y recomposiciones territoriales y socio-políticas, editado por Elkin Velásquez y Henri Godard, 27 - 35. Bogotá: Universidad Externado de Colombia, 2008.

Ziiccardi, Alicia (coord.). Participación ciudadana y políticas sociales del ámbito local. México D.F.: Universidad Nacional Autónoma de México, Instituto de Investigaciones Sociales, Consejo Mexicano de Ciencias Sociales, 2004.

\section{Legislación}

Congreso de la República de Colombia. Constitución Política de la República de Colombia. Bogotá: Congreso de la República de Colombia, 1991. 
Congreso de la República de Colombia. Ley 30 de 1992 por el cual se organiza el servicio público de la educación superior en Colombia. Bogotá: Congreso de la República de Colombia, 1992.

Congreso de la República de Colombia. Ley 115 de 1994. Ley General de Educación. Bogotá: Congreso de la República de Colombia, 1994.

Congreso de la República de Colombia. Ley 1.346 del 31 de julio de 2009. Por medio de la cual se aprueba la Convención sobre los derechos de las personas con discapacidad adoptada por la Asamblea General de las Naciones Unidas el 13 de diciembre de 2006. Bogotá: Congreso de la República de Colombia, 2009.

Congreso de la República de Colombia. Ley Estatutaria 1.618 del 27 febrero de 2013 por medio de la cual se establecen las disposiciones para garantizar el pleno ejercicio de los derechos de las personas con discapacidad. Bogotá: Congreso de la República de Colombia, 2013.

Consejería Presidencial para la Política Social (CPPS) y Corporación Andina de Fomento (CAF). Política pública en discapacidad. Metodología para la formación. Bogotá: Presidencia de la República, 2002.

Departamento Nacional de Planeación (DNP) y Consejo Nacional de Política Económica y Social (CONPES). Política pública nacional de discapacidad. Documento CONPES Social 80. Bogotá: República de Colombia, 2004.

Departamento Nacional de Planeación (DNP) y Consejo Nacional de Política Económica y Social (CONPES). Política pública nacional de discapacidad e inclusión social. Documento CONPES Social 166. Bogotá: República de Colombia, 2013.

\section{Bases de datos}

Departamento Administrativo Nacional de Estadística (DANE). Censo general 2005. Discapacidad. Personas con limitaciones permanentes. Departamento Administativo Nacional de Estadística. Bogotá: 2005. https://www.dane.gov. co/files/censo2005/discapacidad.pdf (último acceso: marzo de 2015).

Ministerio de Protección Social (MPS). Registro de localización y caracterización de personas con discapacidad. 20ll. http://www.minsalud.gov.co/proteccionsocial/Paginas/DisCapacidad_RLCPD.aspx (último acceso: 9 de junio de 2014). 